American Journal of Applied Sciences 9 (6): 894-901, 2012

ISSN 1546-9239

(C) 2012 Science Publications

\title{
A New Method to Correlate a Possible Coupling between the Upper and the Lower Atmosphere
}

\author{
${ }^{1}$ Wayan Suparta and ${ }^{2}$ Grahame J. Fraser \\ ${ }^{1}$ Institute of Space Science (ANGKASA), \\ University Kebangsaan Malaysia, 43600 Bangi, Selangor Darul Ehsan, Malaysia \\ ${ }^{2}$ Department of Physics and Astronomy, University of Canterbury, Christchurch, New Zealand
}

\begin{abstract}
Problem statement: Sun-earth coupling studies are one of the methods of projection to explain certain physical mechanisms of how solar activity can influence weather/climate changes. Approach: To gain insight of the above challenge, analysis of propagation delays in GPS-transmitted signals in the upper and lower atmosphere during transient events associated with intense geomagnetic storms was conducted within the Antarctic polar cap. Results: An increase in Total Electron Content (TEC) indicates an increase in the density of energetic particles in the atmosphere and major changes in the signals would affect the water vapor phase transition in the troposphere. Conclusion/Recommendations: From the correlation between the ionospheric TEC and atmospheric Precipitable Water Vapor (PWV) variations, the Global Electric Circuit (GEC) is identified as a possible physical mechanism for the coupling process.
\end{abstract}

Key words: GPS TEC-PWV, upper-lower atmosphere, coupling mechanism

\section{INTRODUCTION}

The Sun is the source of energy that causes the motion of the atmosphere, drives the Earth's climate and controls the weather. Recently, it has been demonstrated that solar radiation and cosmic ray flux variability affect the processes in the middle atmosphere (Lastovicka, 2005). Similar topics have also been discussed in terms of the global atmospheric electric field (Harrison, 2008; Tinsley, 2008). For many decades, demonstrating the direct influence between solar variability and climate change on atmospheric dynamics and their physical mechanisms based on short term data has been proven difficult. One key element that is very often taken as evidence of a response is the similarity of the periodicities between solar activity indices and different meteorological parameters. This similarity, often in the form of coherence, requires analysis of large quantities of data. In addition, the analysis requires improved specification of both the amplitudes and timescales of solar radiative output changes on climatological timescales and the climate sensitivity to small insolation changes (Lean and Rind, 1998). It is repeatedly difficult to obtain significant results in the presence of unrelated periodic variability.

It is well established that variations in solar EUV and Total Solar Irradiance (TSI) radiation impact the thermal structure and composition of the atmosphere but details of the physical mechanisms coupled to the lower atmosphere are still ambiguous. Variations in the galactic cosmic ray intensity, modulated by solar activity, are also manifested in changes in atmospheric ionization. However, there is some uncertainty that these have significant effects on the lower atmosphere (Erlykin et al., 2009). In this study, we have investigated the well-defined driving force of transient Total Electron Content (TEC) enhancement during intense geomagnetic storms and the consequential changes in the Precipitable Water Vapor (PWV) based on the study by Suparta et al. (2008). The suggestion introduced here in the analysis of solar influence on water vapor is novel and is believed to put across a new perspective on how solar activity can influence climate change.

The basis of the present study on the upper-lower atmospheric coupling was reported by Suparta et al. (2008) who have studied the high energy transient events associated with the intense solar flares of October/November 2003, using TEC as a measure of solar activity and PWV as the terrestrial response. Although the GPS receivers were originally installed for geophysical and geodetic applications, they have been employed to accurately measure the atmospheric PWV and the ionospheric TEC at fine temporal and spatial resolution. Both quantities have been estimated from the refractive index changes integrated along the

Corresponding Author: Wayan Suparta, Institute of Space Science (ANGKASA), University Kebangsaan Malaysia, 43600 Bangi, Selangor Darul Ehsan, Malaysia Tel: +603 89214729 Fax: +603 89216856 
Am. J. Applied Sci., 9 (6): 894-901, 2012

ray path between the satellites and the receivers. Suparta et al. (2008) found a significant correlation between PWV and TEC during four geomagnetic storms but there was no significant correlation in the absence of storms. Given the significant correlation between PWV and TEC, the possible mechanisms involved in the PWV response to the solar activity (shown in TEC) are discussed in section results and discussion.

\section{MATERIALS AND METHODS}

One of the established Global Navigation Satellite Systems (GNSS) for space-based systems is the US Global Positioning System (GPS). The current constellation of this system consists of 31 satellites in six orbits with an inclination of $55^{\circ}$ (Kaplan and Hegarty, 2006; Parkinson and Spilker, 1997). The GPS satellites transmit in the radio range at L-band frequencies and have two modulated frequencies of 1575.42 MHz as $\mathrm{L}_{1}$ and $1227.6 \mathrm{MHz}$ as $\mathrm{L}_{2}$. The orbit of each GPS satellite is approximately circular and semisynchronized and is placed at $20,183 \mathrm{~km}$ from the mean surface of the Earth with orbital radius of $26,600 \mathrm{~km}$. This constellation produces global coverage of 24 hours per day. Based on these orbital characteristics, TEC and PWV measurements from the ground GPS receivers can easily detect any temporal variations within the ionosphere and troposphere, respectively. By employing a high precision GPS receiver with excellent antenna design that is capable of eliminating the multipath effects, the main error of the GPS signals is expected to come only from the nature of the ionosphere and troposphere layers. Figure 1 shows the ray path of the GPS signals, together with their major effects during propagation in the atmosphere.

When the GPS signal propagates through the Earth's atmosphere, it is significantly affected by the variability of the refractive index of the ionosphere and troposphere. This causes an excess delay of the signal and the changes in the refractive index with the height causes bending of the signal. The total delay along the slant path is composed of two parts: ionospheric delay and tropospheric delay. The ionospheric delay is frequency dependent and it can be nearly corrected by observing both frequencies transmitted by the GPS satellites $\left(\mathrm{L}_{1}\right.$ and $\left.\mathrm{L}_{2}\right)$ using a dual-band GPS receiver (Kunches and Klobuchar, 2000). The ionospheric delay effect in the GPS signals can also be minimized during the processing by adopting the Precise Point Positioning (PPP) strategy, as implemented in the JPLGIPSY-OASIS software (Zumberge et al., 1998). The remaining delay is the tropospheric delay or neutral delay that is not frequency dependent, but depends on the constituents of the atmosphere. As such, this delay along the path of the radio signal to a receiver on the ground is sensitive to local surface pressure, temperature and water vapor variations. Additionally, by differencing the pseudorange at two different frequencies, the errors caused by clock biases between the receiver and satellite or non-dispersive components in the tropospheric delay calculation can be isolated. Based on the ray paths illustrated in Fig. 1, both TEC and PWV values have been determined as presented in the following sections.

GPS TEC determination: Line-of-sight or Slant TEC (STEC) measurements along the path from the satellite to the receiver were determined from both the pseudorange (code) and the carrier phase at dualfrequencies. Both measurements contain noise and bias due largely to multiple paths and are subject to cycle slips associated with rapid ionospheric scintillations. STEC values were quantified from a ground-GPS receiver around the ionospheric pierce point $(\sim 350 \mathrm{~km}$ for poles, or $\sim 400 \mathrm{~km}$ for equator). STEC was then converted into Vertical TEC (or TEC for simplicity) by using the modified single-layer model (MSLM) mapping function by approximating the JPL extended slab mapping function. To obtain the final precise TEC estimation, the inherent instrumental biases from both the receiver and the satellites were reduced and corrected by using the Differential Code Bias (DCB) obtained from the AIUB Data Center of Bern University, Switzerland (ftp://ftp.unibe.ch/aiub/CODE/). Details of the method for calculating the absolute TEC by employing a dualfrequency code and phase measurements can be found elsewhere (Hofmann-Wellenhof et al., 2001).

GPS PWV determination: The PWV total was calculated from the GPS signals and the surface meteorological data. Surface meteorological measurements were used to correct the errors caused by atmospheric delays of GPS transmissions. Deriving the PWV from the GPS observations involved five steps. First, the total tropospheric delay was estimated by constraining the positions of widely-spaced GPS receivers and measuring the apparent error in a position every 30s. When all system related errors have been accounted for, the residual error is presumed to come only from the neutral atmosphere (Fig. 1). Second, the total signal delay measured by the GPS receiver from all satellites in view were mapped onto the zenith direction using Vienna hydrostatic appropriate mapping function (VMF1) and combined to give the Zenith Total Delay (ZTD). 
Am. J. Applied Sci., 9 (6): 894-901, 2012

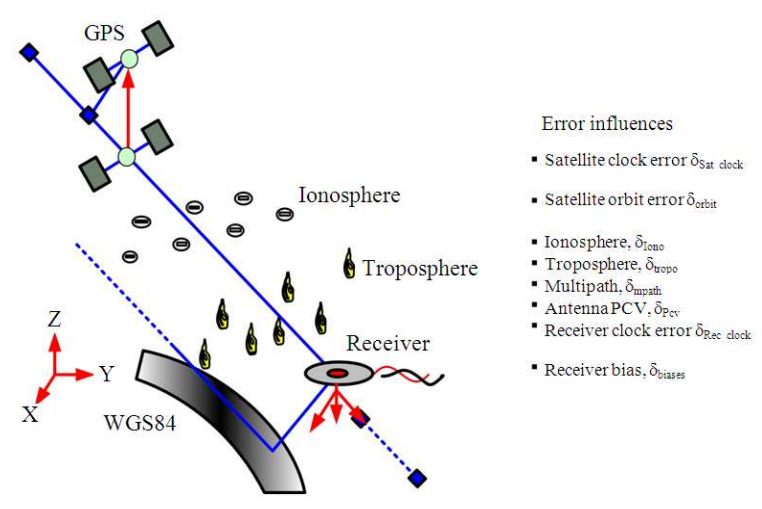

Fig. 1: Ray path of GPS signals that propagates through the Earth's atmosphere

In addition to the precise ZTD estimation accuracy, the residual tropospheric errors were then estimated as a part of the adjustment process. Alternatively, the ZTD in this study was calculated based on the Modified Hopfield model. Third, the Zenith Hydrostatic Delay (ZHD) was calculated based on Saastamoinen model which uses the surface pressure measurement and precise geographic location of the GPS receiver. Fourth, the Zenith Wet Delay (ZWD) was obtained by subtracting the ZHD from ZTD. Finally, PWV was derived from ZWD signals and a conversion factor that was proportional to the weighted mean surface temperature. The mean air temperature was estimated from the surface temperature measurement at the site. Detailed of the calculation of the GPS derived PWV for this study can be found in the study of Suparta et al. (2008). In this study, the Tropospheric Water Vapor Program (TroWav) written in Matlab was used to produce the actual PWV data.

Data analysis: In the proposed TEC-PWV coupling mechanism, solar activity is assumed to have a direct and clear influence on TEC. With this assumption, the indirect influence of solar events on PWV by correlating ionospheric TEC with PWV variations are practicable. The assumption is also valid because both the ionospheric and the tropospheric layers are closely coupled, continuously interacting and both layers in some ways affect GPS signals. The two components are also connected by the global atmospheric electric circuit (Tinsley, 2008). To best characterize the solarclimate relationship based on TEC-PWV approach, a strong event is preferred as it will minimize the influence of unwanted instrumental or geophysical noise. The strong event in this subject is a geomagnetic storm. For that purpose, the primary data used in this study are GPS and the surface meteorology data.
For the short-term correlation between TEC and PWV, the percentage deviation of TEC (\% $\%$ TEC) was employed rather than the absolute TEC. This approach provided the TEC deviation (enhancement or depletion) from the mean value in response to the solar forcing in the ionosphere. On another note, the percentage of TEC and the PWV results for the case of major geomagnetic storms, observed in Antarctica were used to demonstrate the coupling process between the upper and the lower atmosphere. On this basis, the percentage of TEC was calculated by separating the actual TEC with the TEC value measured during the quiet day and this yielded the storm affected-days. Solar and geomagnetic activity (sunspot number (SSN) and solar flux $\left(\mathrm{F}_{10.7}\right)$, Interplanetary Magnetic Field (IMF) and geomagnetic activity through the Disturbance Storm time (Dst) and cosmic ray) were also presented to indicate their relationship with the GNSS results. The investigations focused on the October/November of 2003 geomagnetic storm which covers the period from 28 October to 1 November 2003.

\section{RESULTS AND DISCUSSION}

The analysis and proposed method for the coupling process are presented in the following sections.

Changes in TEC and PWV: Figure 2 shows the TEC variations for several stations in Antarctica during the geomagnetic storm of October/November 2003 (socalled the Halloween storm). As shown in the figure, Scott Base (SBA: $77.85^{\circ} \mathrm{S}, 166.76^{\circ} \mathrm{E}$ ) has been plotted from its original value, while stations above and below the SBA have been shifted each by \pm 150 . As a result (Fig. 2a), Sanae (VESL: $71.67^{\circ} \mathrm{S}, 02.83^{\circ} \mathrm{W}$ ) and Macquarie Island (MAC1: $\left.54.50^{\circ} \mathrm{S}, 158.93^{\circ} \mathrm{E}\right)$ showed a similar $\mathrm{x}$-axis, indicating that the TEC value at MAC1 was twice higher than at VESL. From the average percentage of TEC on October/November of 2003 (Fig. $2 \mathrm{~b}$ ), an abnormal peak with values of 33 TECU was found. Daily variation of TEC in normal condition (in the absence of storm) showed a diurnal pattern. It was higher at around noon local time and lower when the sun was inactive (so-called the Fountain effect). On the other hand, during the geomagnetic storm, the GPS signals in the ionosphere were clearly influenced by solar activity (so-called the upper atmosphere effects). From the figure, it can be clearly observed that the TEC signal at all the stations showed obvious disruption as indicated by the grey background. The signal is very sensitive compared to the event before and after the storm.

From the viewpoint of the lower atmosphere (shown by PWV), a disturbance appeared in Fig. 3. 
Am. J. Applied Sci., 9 (6): 894-901, 2012

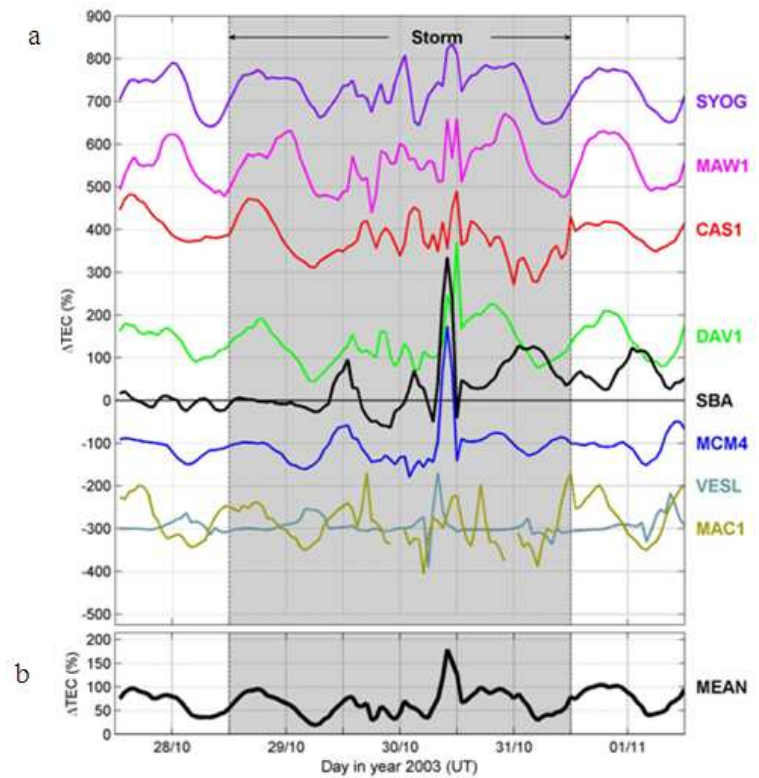

Fig. 2: TEC variability measured over Antarctica for the geomagnetic storm of October/November 2003. SBA is used as a reference and those above and below this stations are shifted by \pm 150

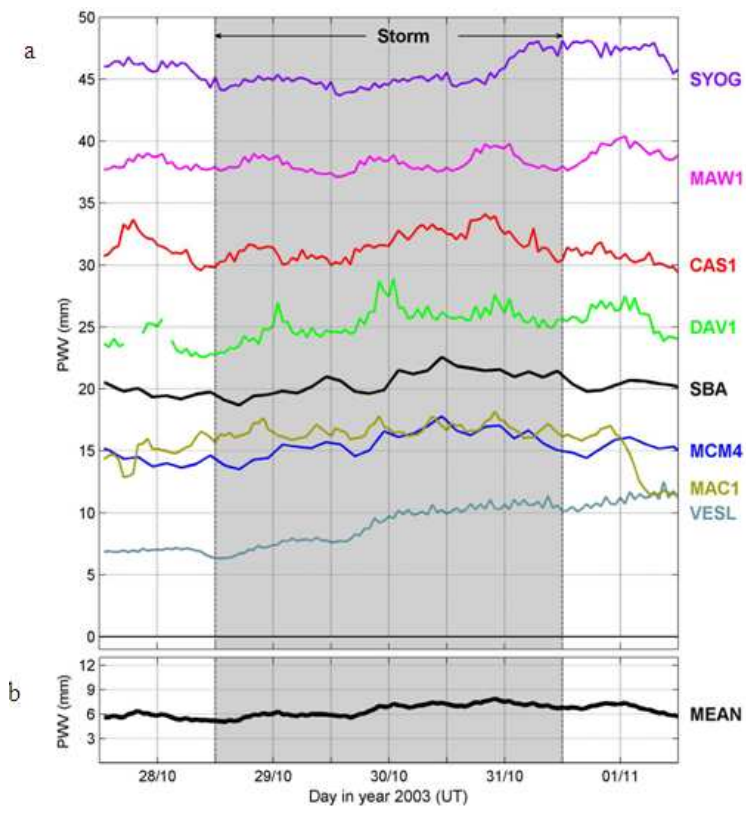

Fig. 3: PWV changes measured over Antarctica during the geomagnetic storm of October/November 2003. Note that the PWV value for MAC1 is used as a reference and the others were offset every $+5 \mathrm{~mm}$ to show their trends
Although the disturbance was not as strong as the effect of the upper atmosphere (please refer to Fig. 3a), it can be clearly seen that during major geomagnetic storms, the PWV content increased by approximately $1.52 \mathrm{~mm}$. The increase in PWV is equivalent to $9.8 \mathrm{~mm}$ ZTD delay. Note that the overall PWV average is $6.4 \mathrm{~mm}$ (Fig. 3b). As can be seen in Fig. 3a, there are four stations that are a pair (sharing similarity), the SBAMCM4 (similar trend and the response is close to the percentage of TEC), SYOG-VESL (increased after the storm), DAV1-MAW1 (oscillating or diurnal pattern) and MAC1-CAS1 (increased during the storm). These differences in the trend lead to a complex interpretation. The differences in the trend perhaps are caused by daily geographic variations between the paired stations. For instance, SBA-MCM4 has a higher geographic position of about $10^{\circ}$ latitude compared to DAV1-MAW1. The presence of high PWV variations at each station was also affected by seasonal variations, where the month of geomagnetic storms observed coincided with the seasonal transition from Austral spring to Austral summer. On the other hand, the seasonal variation of PWV in this region seems to be most directly controlled by the thermodynamic of the lower atmosphere, where the surface temperatures and solar radiation contribute significantly (Suparta et al., 2009; 2010).

A possible link between upper and lower atmosphere: To analyze a possible coupling process during periods of high geomagnetic activities (highest energy), the influence of solar activity on the lower atmosphere is depicted in Fig. 4. Note that all values plotted in the figure have been averaged from all stations used in this study, except for the solar Cosmic Ray (CR) which has been obtained from McMurdo (MCM4) and South Pole (SPO). The SSN and $\mathrm{F}_{10.7}$ in Fig. 4a and Solar Wind (SW) conditions represented by the southward component of the interplanetary magnetic field (IMF $B_{z}$ ) from ACE spacecraft (Fig. 4b) have been used as the solar activity. The storm is characterized by Disturbance Storm time (Dst) in Fig. $4 c$, obtained from WDC2-Kyoto, Japan. Ionospheric TEC (Fig. 4e) derived from the GPS signals are the subject that represents the upper atmospheric variation. As the GPS signals propagate through the atmosphere before reaching the receiver on the ground, the CR variation (Fig. 4e) is a good candidate to acts as an intermediate in the transfer of energy from the upper to the lower atmosphere. PWV and surface temperature (in Fig. $4 \mathrm{f}$ and $4 \mathrm{~g}$ ) represent the lower atmosphere or the terrestrial climate response. 
Am. J. Applied Sci., 9 (6): 894-901, 2012

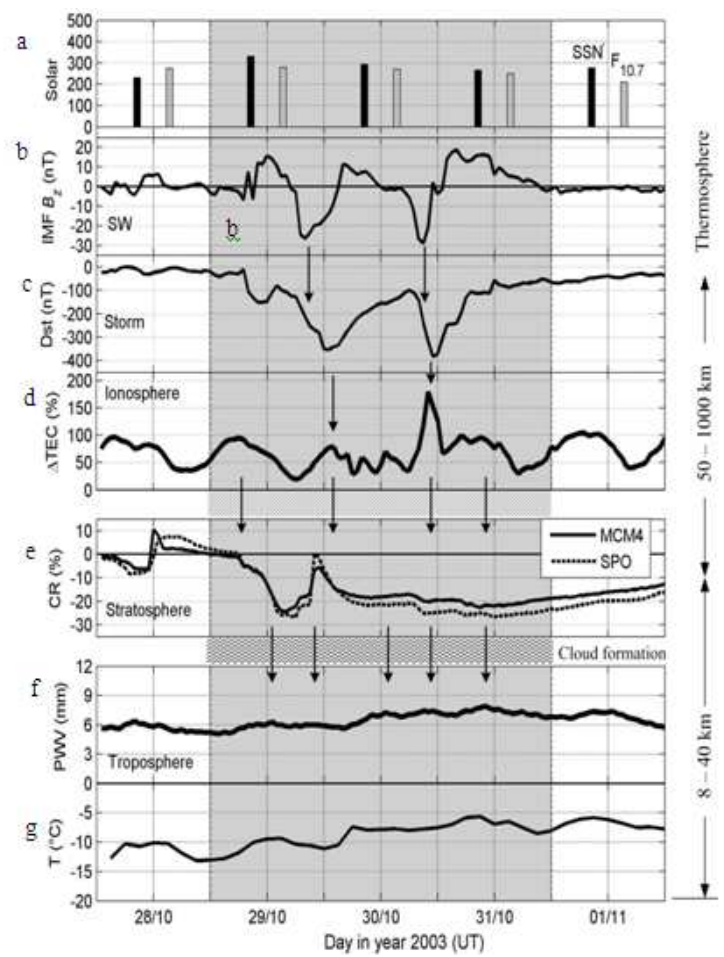

Fig. 4: Details of the solar influence on the lower atmosphere during a period of high geomagnetic storm at high latitudes (altitude of the atmospheric layers is not scaled). Downward arrows show the energy transfer from the upper to the lower atmosphere

As shown in the figure, $\mathrm{SSN}$ and $\mathrm{F}_{10.7}$ values are more than 200 , indicating solar activity is very active. These activities are followed by strong negative polarity of IMF $B_{z}$ of less than $-25 n$ nT causing a decrease in the geomagnetic activity that is correlated with negative peak of Dst. Two episodes of Dst (with two valley peaks) which were less than $-100 \mathrm{nT}$ implies that a major geomagnetic storm growth phase had developed. In that respect, solar and geomagnetic activity correlated well with TEC variation. For this strong relationship, a reasonable coupling of the Sunearth system can be expected. When the IMF $B_{z}$ and Dst move southwardly, the TEC will suddenly increase indicating that geomagnetic storm plays an important role in communication systems. During periods of high geomagnetic activities (highest energy), the interaction between solar activity and TEC correlated well (e.g., Tsurutani et al. (2006). In the middle atmosphere (stratosphere), the coupling process looks simple but shows that the manifestation of solar effects in the middle atmosphere is complex. Ionization in the lower part of the atmosphere is almost exclusively produced by Galactic Cosmic Rays (GCRs) where the clouds reflect more energy than they trap and this leads to cooling. This process can be seen in the second episode of Dst which diminishes the CR peak. Looking at the lower atmosphere, it is clearly shown that the PWV variation is very close to the surface temperature. The surface temperature and pressure are the biggest contributing factors in changing the PWV content (Suparta et al., 2008; 2009). On the other hand, the PWV response to the upper atmosphere (shown by TEC) has a lag of about $4 \sim 6 \mathrm{~h}$ (on average).

Specific to the upper and the lower atmospheric interactions, the increase in TEC indicates substantial disturbances to the global atmospheric circuit. The TEC increase has been found to be caused by the higher density incidence of energetic particles in the atmosphere governed by storms and the major change in the conductivity in and above the ionospheric Dregion. This brings consequences to changes in surface temperature and pressure in the lower atmosphere and PWV as well. Both these parameters are susceptible to changes in the global atmospheric electric circuit. Burns et al. (2007) have described statistically of the significant changes in surface pressure with changes in the vertical electric field at Vostok station in Antarctica. The consequential changes can also be expected in cloud structure (e.g., review by Tinsley (2008), including cloud thickness (Harrison, 2008) and cloud base temperature at a middle latitude station during a solar flare (accompanied by a change in downward long-wave radiation) (Harrison and Ambaum, 2008).

Proposed physical mechanism: Figure 5 illustrates the method of the TEC-PWV approach proposed. The coupling illustrated by the dashed line ("Indirect") is to be the global atmospheric electric circuit. Referring to Fig. 5, the Sun's effects on the Earth's magnetosphere, ionosphere and in the atmospheric dynamics, particularly on the Earth's magnetic field lead to an important event, known as the geomagnetic storm. This storm tends to occur during times of geophysical disturbances resulting from the increase in solar activity. The sources of changes are in solar irradiance through sunspot and faculae, solar flare frequency and intensity and changes in the various coronal phenomena communicated via solar wind (Fuller-Rowell et al., 1994; Hathaway and Wilson, 2004). The transfer of energy from the Sun to the magnetosphere by solar wind is controlled by the electric fields. The Earth's magnetic field acts as wires to relay the energy from the solar wind to the ionosphere in the form of heat (Lyon, 2000). 


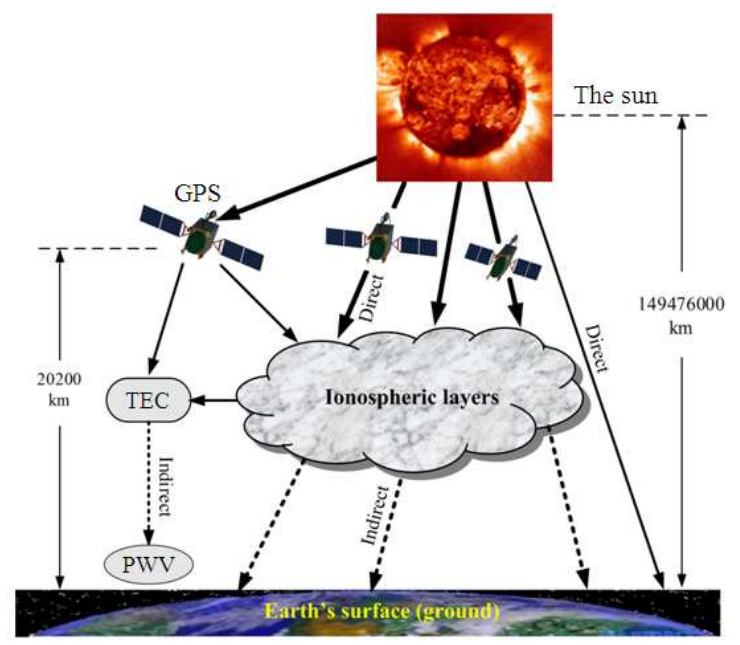

Fig. 5: The GPS TEC-PWV approach

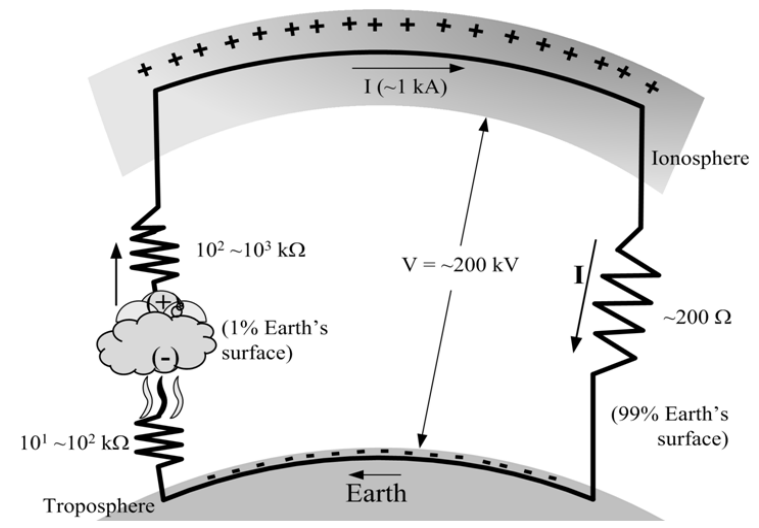

Fig. 6: The upper-lower atmospheric coupling mechanism through a global electric circuit role

Thus, the key mechanism in the coupling of the solar wind with the magnetosphere is the reconnection (Dungey, 1961). The coupling sets up a large system of magnetic-aligned currents (FACs). The change of current (energetic particle) during extreme solar activities flowing through the ionosphere and its change with time impact the Earth's atmosphere as well as the communication system between the layers. Since solar activity affects GPS signals, the disturbances will act as a medium to transfer energy for the process of ionosphere-troposphere coupling.

To clarify the proposed upper-lower atmospheric coupling through TEC-PWV approach, Fig. 4 and 5 are merged into the form of Global Electric Circuit (GEC) as shown in Fig. 6. The GECs are shown to be organized by lightning phenomena over the geomagnetic equator. The expected value in the circuit is estimated on the surface during a fair weather condition. The negative charge seen in the figure is transferred to the Earth (troposphere), while the positive charge above the thundercloud is transferred to the conducting upper atmosphere and form a loop for the coupling process. As shown on the left of the figure, global lightning activity charges the ionosphere and the current returns into the ground through fair weather conduction. This event forces the water vapor through thermal heating and transports energy between the ionosphere and troposphere layers. The electrical discharge is believed to be from the lightning-induced electron precipitation events that could propagate downwards from clouds to the troposphere. During this event, a substantial disturbance from the upper troposphere until the ionospheric E-region (affecting the E-sporadic layer) is indicated by the increase of TEC. During these transport processes, a diurnal TEC cycle develops due to the diurnal rotation of the Earth. These effects are expected to be greater at high latitudes due to the enhanced incident of particles transported along the geomagnetic field lines. Consequently, the PWV will change following the seasonal TEC changes (Suparta et al., 2008; 2010).

At high latitudes, increase of altitudinal redistribution of condensation nuclei occurs under increased electric field current of the atmosphere. This process is modulated by cosmic rays resulting in water vapor condensing in an area where the nuclei concentration is small. With high modulation of cosmic ray, water vapor will increase as a consequence of phase transition of water vapor and cloud formation. The cloud leads to changes in radiation balance, reduced radiative cooling and altered thermo-baric field (temperature, $\mathrm{T}$ and pressure, $\mathrm{P}$ ) in the troposphere (Fig. 4, bottom). On the other hand, the global atmospheric electric field provides a clear physical link between TEC in the ionosphere and PWV near the surface, especially at high latitudes during the occurrence of intense solar flares. These mechanisms suggest that the temporal distribution and strength of solar activity as indicated by geomagnetic disturbances and ionospheric activity are important input for seasonal cycle of water vapor. This approach from the GPS perspective is applicable in correlating ionospheric TEC and PWV. It should be noted that TEC and water vapor are clearly two different atmospheric components, but both traversed the GPS signals. Therefore, in this method, TEC is not directly controlling the PWV. It is only used as an indicator of solar influences on the terrestrial climate. 


\section{CONCLUSION}

This study has successfully demonstrated the use of TEC-PWV approach to correlate the upper and the lower levels of the atmosphere during a major geomagnetic storm associated with a very active period in October/November 2003. The energy transfer from the solar activity to the Earth's surface is shown to be the global atmospheric electric circuits over the geomagnetic equator. The coupling mechanism proposed from the GPS observations which is an increase in TEC indicates substantial disturbances to the global electric circuit. Thus, the coupling process can be explained as follows. An increase in the incidence density of the energetic particles in the atmosphere and a major change in the conductivity from the ionospheric E-region to the lower atmosphere with changes of cloud structure and cloud base temperature by cosmic ray modulation (accompanied by a change in downward long-wave radiation) and an increase in TEC will force the PWV through the water vapor phase transition and thermo-baric changes in the troposphere. These effects during the intense geomagnetic storm at high latitudes are expected to be greater due to the enhanced incident of particles transported along the geomagnetic field lines.

\section{ACKNOWLEDGEMENT}

This study was supported by the Ministry of Higher Education Malaysia (MOHE) under UKM-LL07-FRGS0211-2010 grant. The author would like to express gratitude to the Academy of Sciences Malaysia under PKT 1/2003 grant through Ministry of Science, Technology and Innovation Malaysia (MOSTI), Antarctica New Zealand (ANZ) and National Institute of Water and Atmospheric Research Ltd., New Zealand (NIWA) for supporting this research. We would also like to thank the Solar Influences Data analysis Center (SIDC), Royal Observatory of Belgium for the sunspot data, D.J. McComas at SWRI and CDAWeb for providing the ACE/SWEPAM Solar Wind Experiment Data and the World Data Center (WDC)-C2, Kyoto, Japan for the hourly final of Dst data, the Scripps Orbit and Permanent Array Center (SOPAC) and John W. Bieber of Bartol Research Institute for providing the cosmic ray data in Antarctica.

\section{REFERENCES}

Burns, G. B., B.A. Tinsley, A.V. Frank-Kamenetsky and E.A. Bering, 2007. Interplanetary magnetic field and atmospheric electric circuit influences on ground-level pressure at Vostok. J. Geophys. Res., 112: D04103-D04103. DOI: 10.1029/2006JD007246
Dungey, J.W., 1961. Interplanetary magnetic field and the auroral zones. Phys. Rev. Lett., 6: 47-48. DOI: 10.1103/PhysRevLett.6.47

Erlykin, A.D., G. Gyalai, K. Kudela, T. Sloan and A.W. Wolfendale, 2009. Some aspects of ionization and the cloud cover, cosmic ray correlation problem. J. Atmos. Sol-Terr. Phys., 71: 823-829. DOI: 10.1016/j.jastp.2009.03.007

Fuller-Rowell, T.J., M.V. Codrescu, R.J. Moffett and S. Quegan, 1994. Response of the thermosphere and ionosphere to geomagnetic storms. J. Geophys. Res., 99: 3893-3914. DOI: 10.1029/93JA02015

Harrison, R.G. and M.P.H. Ambaum, 2008. Enhancement of cloud formation by droplet charging. Proc. Royal Soc. A., 464: 2561-2573. DOI: 10.1098/rspa.2008.0009

Harrison, R.G., 2008. Discrimination between cosmic ray and solar irradiance effects on clouds, and evidence for geophysical modulation of cloud thickness. Proc. R. Soc. A., 464: 2575-2590. DOI: 10.1098/rspa.2008.0081

Hathaway, D.H. and R.M. Wilson, 2004. What the sunspot record tells us about space climate. Sol. Phys., 224: 5-19.

Hofmann-Wellenhof, B., H. Lichtenegger and J. Collins, 2001. Global Positioning System: Theory and Practice. 5th Edn., Springer Verlag, Wien, New York, ISBN: 3211835342, pp: 382.

Kaplan, E.D. and C.J. Hegarty, 2006. Understanding GPS: Principles and Applications. 2nd Edn., Artech House, Boston, Mass., ISBN: 1580538940, pp: 703.

Kunches, J.M. and J.A. Klobuchar, 2000. Eye on the ionosphere, some aspects of the variability of geomagnetic storms. GPS Solutions, 4: 77-78.

Lastovicka, J., 2005. Preface. J. Atmos. Solar-Terr. Phys., 67: 1-1.

Lean, J. and D. Rind, 1998. Climate forcing by changing solar radiation. J. Clim., 11: 3069-3094. DOI: $\quad 10.1175 / 1520-$ 0442(1998)011<3069:CFBCSR>2.0.CO;2

Lyon, J.G., 2000. The solar wind-magnetosphereionosphere system. Science, 288: 1987-1991. PMID: 0010856203

Parkinson, B.W. and J.J. Spilker Jr., 1997. Global Positioning System: Theory and Applications. 1st Edn., American Institute of Aeronautics and Astronautics, Washington, D.C., ISBN: 156347106X, pp: 793.

Suparta, W., Z.A. Abdul Rashid, M.A. Mohd Ali, B. Yatim and G.J. Fraser, 2008. Observations of Antarctic precipitable water vapor and its response to the solar activity based on GPS sensing. J. Atmos. Sol-Terr Phys., 70: 1419-1447. DOI: 10.1016/j.jastp.2008.04.006 
Suparta, W., M.A. Mohd. Ali, B. Yatim and G.J. Fraser, 2009. Analysis of GPS-sensed atmospheric water vapour variability and its response to the terrestrial winds over Antarctica. J. Phys. Chem. Earth, 34: 72-87.

Suparta, W., B. Yatim and M.A.M. Ali, 2010. Solar forcing on Antarctic terrestrial climate: A study by means of GPS observations. Acta Geophys., 58: 374-391. DOI: 10.2478/s11600-009-0035-4

Tinsley, B.A., 2008. The global atmospheric electric circuit and its effects on cloud microphysics. Rep. Prog. Phys., 71: 066801-066801. DOI: $10.1088 / 0034-4885 / 71 / 6 / 066801$
Tsurutani, B.T., A.J. Mannucci, B. Iijima, F.L. Guarnieri and W.D. Gonzalezet et al., 2006. The extreme Halloween 2003 solar flares (and Bastille Day, 2000 Flare), ICMEs, and resultant extreme ionospheric effects: A review. Adv. Space Res., 37: 1583-1588. DOI: 10.1016/j.asr.2005.05.114

Zumberge, J.F., M.B. Heflin, D.C. Jefferson, M.M. Watkins and F.H. Webb, 1998. Precise point positioning for the efficient and robust analysis of GPS data from large networks. J. Geophys. Res., 102: 5005-5017. DOI: 10.1029/96JB03860 